\title{
Problems of Birds Sex Determination
}

\author{
Antonina V. Trukhina, Aleksandr F. Smirnov \\ Department of Genetics and Biotechnology, St. Petersburg State University, St. Petersburg, Russia \\ Email: trukhina ant@mail.ru, afsmirnov@bio.pu.ru
}

Received 19 August 2014; revised 10 September 2014; accepted 30 September 2014

Copyright (C) 2014 by authors and Scientific Research Publishing Inc.

This work is licensed under the Creative Commons Attribution International License (CC BY).

http://creativecommons.org/licenses/by/4.0/

(c) (i) Open Access

\begin{abstract}
Sex determination system in birds is characterized by a homo-(Neognatae) and heteromorphic (Paleognatae) sex chromosomes. Heterogametic sex is female (ZZ/ZW system). DMRT1 gene is a gene regarded as a main male sex determining factor in this group of animals. The question remains about the participation of other factors (HEMOGEN, AMH etc.) in appearance of testis, and the role of steroid hormones in formation of ovaries. Complete sex inversion is not typical for species with genotypic sex determination (GSD), although the effect of estrogen metabolites is noted for birds. For birds epigenetic mechanisms of regulation (methylation of DNA and non-coding RNA) have been described for sex controlling genes such as CYP19A1 and DMRT1.
\end{abstract}

\section{Keywords}

\section{Bird, Sex Determination, Epigenetics, Dosage Compensation}

\section{Introduction}

Similar to all animals in birds there are chromosomal differences between the sexes. There are two Z chromosomes in males, while females have got $\mathrm{Z}$ and $\mathrm{W}$ chromosomes, therefore heterogametic sex is female. This type of definition of sex exists in many species of fishes, reptiles, amphibians, crustaceans and birds [1]. Known case of the presence of two systems of sex determination is $\mathrm{XX} / \mathrm{XY}$ and $\mathrm{ZZ} / \mathrm{ZW}$ within a species in the frog Rana raposa [2]. It is thought that these two systems either have a common evolutionary origin or XY system of mammals arose directly from the ZW system of reptiles [3].

The chicken $\mathrm{Z}$ chromosome is large and gene-rich (similar to the mammalian $\mathrm{X}$ chromosome), while the $\mathrm{W}$ is typically small and largely heterochromatic (similar to the mammalian Y). The chicken Z chromosome is about $80 \mathrm{Mb}$ and is highly conserved among avian. The $\mathrm{Z}$ harbors over 1000 genes [1] [4]. W chromosome has got approximately $44 \mathrm{Mb}$ of repeats and about $10 \mathrm{Mb}$ of unique DNA, which may contain up to 20 active genes (Figure 1). 60\% of the W chromosome DNA sequence consists of repeats such as Xho1 (centromeric region) and EcoR1 families (large region of the long arm and the middle of the short arm). There is also Ssp 1 family 


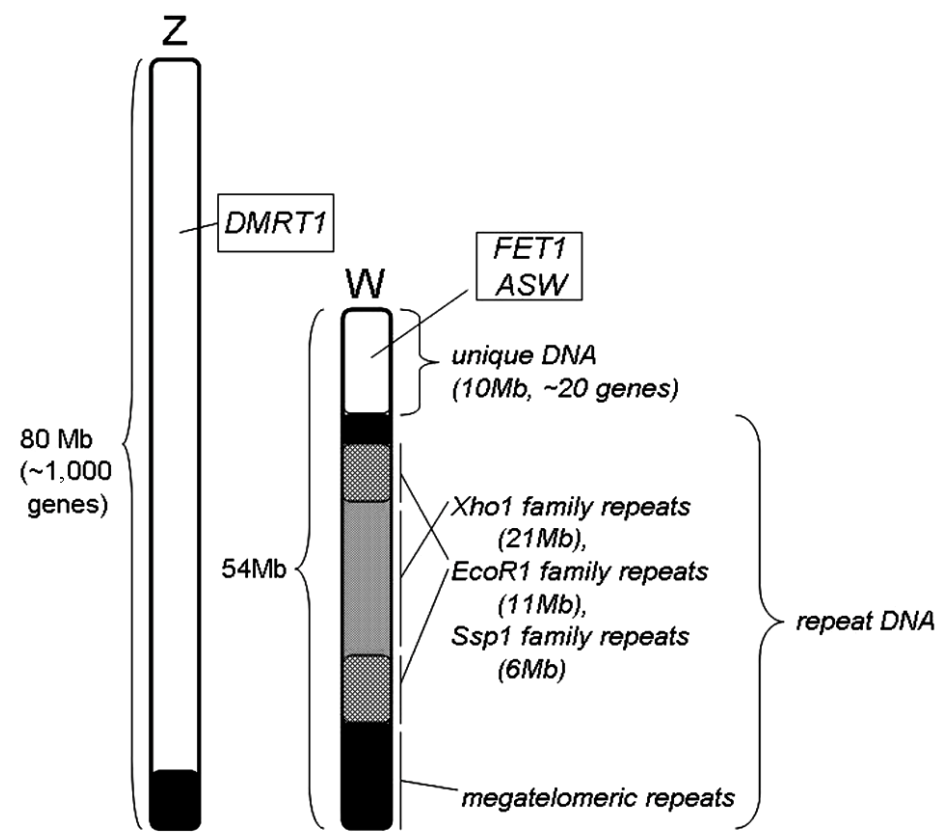

Figure 1. Z and W chromosomes of Gallus gallus domesticus. Euchromatin is showed by white color, repetitive sequences (heterochromatin) is showed by black and gray color. Also there are shown candidate gene for male (DMRT1) and prospective sex determined genes for female (FET1 and ASW).

containing a repeated unit of $0.5 \mathrm{~kb}$, including polypurin/polypyrimidine sequence (GGAGA repeat) at the 3'-end. The sizes of these families are about $6 \mathrm{Mb}$ (Ssp1), $11 \mathrm{Mb}$ (EcoR1) and $21 \mathrm{Mb}$ (Xho1) respectively. Most of the expressed $\mathrm{W}$ genes have open reading frames that are either almost or completely homologous to their Z-linked gametologues. The two exceptions are HINTW, which is divergent from its $\mathrm{Z}$ gametologue, and FAF, which lacks the $\mathrm{Z}$ gametologue. Only $4 \%$ of the chicken $\mathrm{W}$ chromosome DNA sequences have been mapped. According to the most recent release of the chicken genome, this amount is about $1.2 \mathrm{Mb}$ of assembled sequence on the $\mathrm{W}$ [5]-[7]. Importantly, the longest telomere beams of Class 3 (2.8 Mb) is localized to the long arm of the $\mathrm{W}$ chromosome. This phenomenon is associated with recombination hot spots, whose role is to be a specific buffer for gene-rich regions [8].

The number of known genes of $\mathrm{W}$ chromosome expanded to 26. This includes two novel W-linked sequence and three small RNAs reassigned to the chromosome. A number of W-linked genes (with the exception of FAF (Female Associated Factor)) were found to have gametologues on the Z. There was high sequence homology between almost all W- and Z-linked copies at both the DNA level (average 88.4\% identity) and the protein level (average $90.3 \%$ identity). An exception was the gene HINT-W, which showed $41 \%$ sequence and $48.5 \%$ amino acid homology. These results show a clear bias for genes associated with sex and reproduction to be located on the avian $\mathrm{Z}$ sex chromosome, connecting sex determination vith $\mathrm{Z}$ dosage and expression level. For example Z-linked testis-associated genes DMRT1 and HEMOGEN are more highly expressed in the gonads compared to the blastoderm. It is possible that additional Z-linked genes may play a role in sexual differentiation in different tissues through changes in their relative expression [9].

It is thought that sex chromosomes in birds and mammals are not homologous and evolved from different pairs of autosomes. So $\mathrm{Z}$ chromosome of many birds contain homologous regions of chromosome 9 (the most of the 24 namely 17 mapped on chromosome 9), 5, 8, 18, and there is only one site of homology of $\mathrm{X}$ chromosome containing the gene OTC (ㅁnithine transcarbamylase) [4]. The evolution of heterogametic chromosomes $\mathrm{Z}$ and $\mathrm{W}$ is similar to that of $\mathrm{X}$ and $\mathrm{Y}$ in mammals. It is supposed that progressive restriction of recombination between sex chromosomes is an integral feature of chromosomal evolution in female heterogametic. There are three evolutionary strata on the Z chromosome: 132 - 150 (stratum 1), 71 - 99 (stratum 2) and 47 - 57 (stratum 3) million years ago (MYA). It is supposed even older evolutionary stratum 0 which presents on all bird sex chromosomes that contains the dmrt1 gene, but is lacking on any $\mathrm{W}$ homologues. Thus, stratum 0 corresponds to the initial 
differentiated fragment on the bird sex chromosomes and contains the master-switch sex-determining gene of birds [10].

Most Neognathae birds have got very short PAR (Psevdo Autosomal Region) in Z chromosome and it is rather long at Paleognathae [10] [11]. All birds have homologous sex chromosomes that formed about 120 MYA, similarly to the mammalian sex chromosomes (about 165 MYA). However, whereas all mammals and most birds lineages have highly differentiated sex chromosomes, in some groups of birds, such as ratites, the sex chromosomes remain homomorphic [12]. It is believed that sex of these species can be controlled: 1) the W sex chromosome carries a dominant-acting female determinant (a ZZW female bird or a Z0 male bird have not been definitively documented in birds and it has been suggested that such genotypes may be lethal to embryos); 2) sex could be determined by dosage of one or more Z-linked genes, i.e. two copies in males and one in females (a bird with a ZZW genotype develops as a male despite the presence of the $\mathrm{W}$ as would a Z0 bird (with only one Z) that is female); 3) it is possible both of these mechanisms exist [3] [5]. A 3A:ZZZ birds developed as fairly normal males, 3A:ZZW birds developed as intersexes. At hatching, the 3A:ZZW birds had a right testis and a transient left "ovotestis" with both ovarian and testicular structures [13]. Phenotypically female at hatching, the birds eventually lost the ovarian component of the left gonad and they became male at sexual maturity [3]. In 2012 fertile triploid ZZW female of plovers (Charadrius alexandrinus) was described [12] [14]. It points to the special role of $\mathrm{W}$ chromosomes in sex determination in birds. More recently, a breeding female Great ReedWarbler (Acrocephalus arundinaceus) was described that was inferred to be a 2A:ZZW, based on Z microsatellite analysis. These data support the dominant $\mathrm{W}$ hypothesis over $\mathrm{Z}$ dosage. Hence, there is no consistent evidence from these previous studies that clearly supports one hypothesis over the other [3]. Figure 2 presents the data concerned sex of aneuploids birds in terms of the hypothesis of a W dominant gene on sex determining $\mathrm{W}$ chromosome and doze-sensitive of $\mathrm{Z}$ chromosome.

\section{Sexual Differentiation in Birds}

Bird gonads are formed at around embryonic day 3 (E3) and are initially bipotential. Prior to E6 in the male, the Z-linked transcription factor DMRT1 becomes highly expressed in the male gonads, initiating testis development which begins morphologically differentiate at around E6. This may be through regulation of genes such as $S O X 9$ and through repression of female pathways. It leads to the development of bilateral testis, characterized by seminiferous cord structures. Differentiation is mostly complete by E9. In the female Foxl2 becomes expressed at around E5, and this is thought to regulate the expression of the aromatase gene which is important for estrogen synthesis. A second parallel pathway, the R-SPO1/Wnt4 pathways, is also upregulated in female gonads and may play a role in ovarian development which includes fragmentation of the medulla to produce lacunae
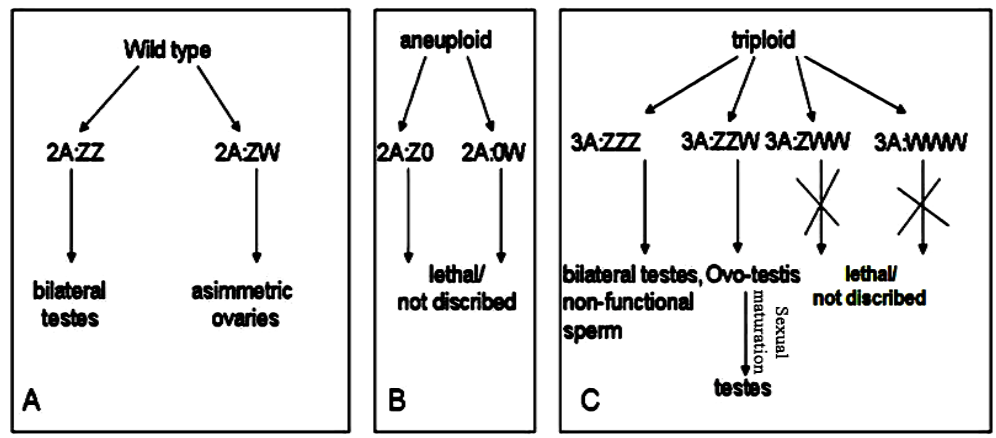

Figure 2. The role of sex chromosome in chicken aneuploids. Study of aneuploids might help to elucidate meaning of an existence of a $\mathrm{Z}$ dosage mechanism or dominant $\mathrm{W}$ mechanism of sex determination that have been suggested for birds. (A) Chickens with normal kariotype have got normally development gonads; (B) Chickens with aneuploidy of sex chromosomes are lethal or not discribed; (C) In triploid male of birds (ZZZ) the bilateral testis are developed normally although sperm was abnormal. ZZW triploid birds were initially female-like and had ovotestis, but the ovarian tissue was regressed with time and these birds presented with more male-like features after sexual maturity [5]. ZWW and WWW triploids are not discribed. 
and proliferation of the cortex cells in the left gonad. Nonproliferation and subsequent regression of the right gonad leads to asymmetric gonads in the female (Figure 3) [5] [6].

It is a very important question about the regulation of genes involved in the control of sex: as DMRT1 and AMH genes are expressed in the gonads of both sexes, but with greater intensity in the males (Figure 4). Dmrt1 is also important in mammalian sex determining gene. Recent studies of Dmrt1 null-mutant XY adult mice have revealed that loss of the Dmrt1 in Sertoli cells causes dramatically decreased expression of several male-specific genes, including Sox9, Sox8, and Ptgds (Prostaglandin-H2 D-isomerase), and significantly robust expression of female-promoting genes Foxl2, Esr1/2, Wnt4 and Rspo1 [15]. DM-genes including Dmrt1 are specifically

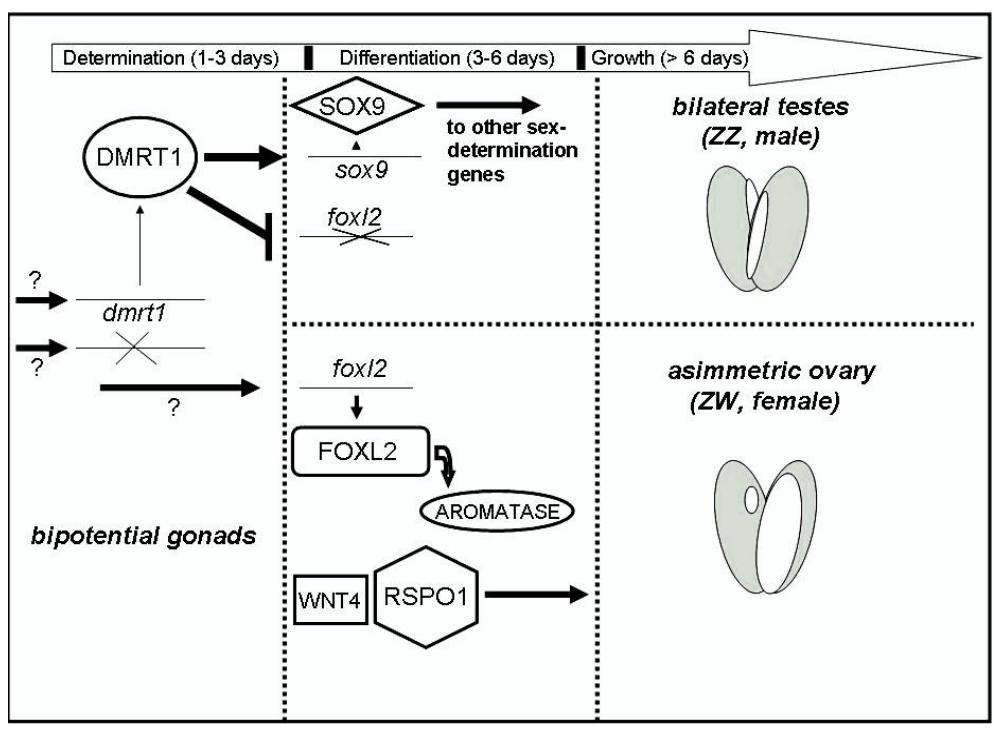

Figure 3. Assumed molecular mechanisms underlying gonad sex development in the chick embryo.

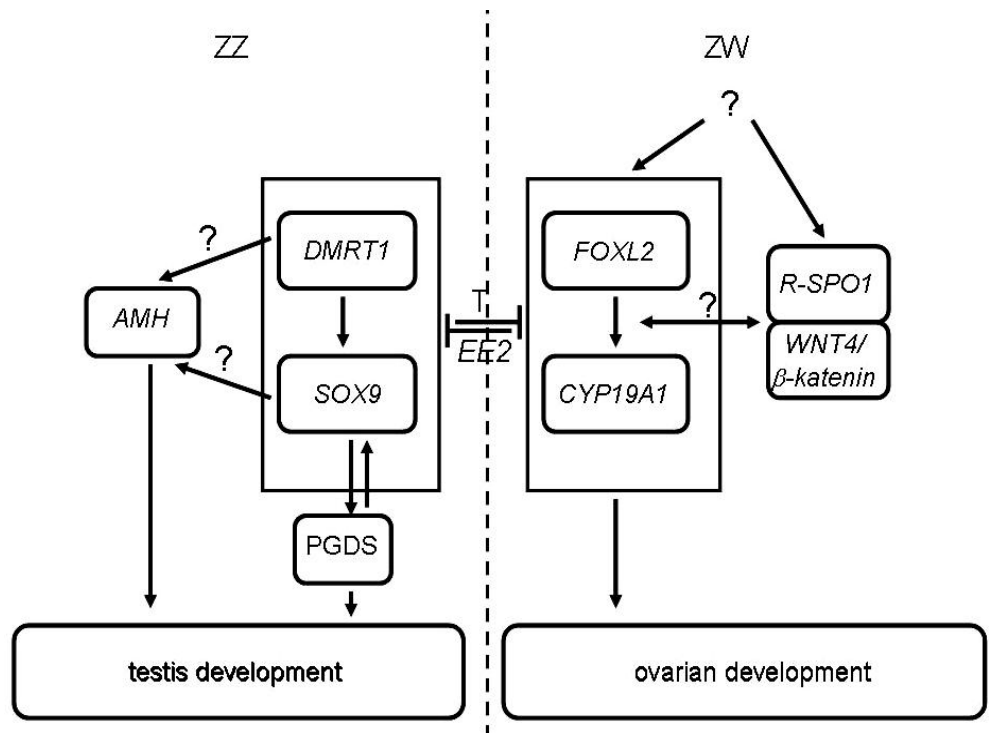

Figure 4. Scheme of interaction of genes involved in the determination of testes and ovaries in chicken. The $D M R T 1$ gene probably indirectly activates expression of SOX9 which is crucial for the emergence of testes jointly with $A M H$. In females $R$-SPO1 gene leads to activation Wnt $4 / \beta$-catenin path and jointly with FOXL2/aromatase leads to differentiation of ovaries. DMRT1 and FOXL2 may exercise antagonizing differentiation testes and ovaries as in mammals. 
expressed in the developing gonads of almost all animals, including vertebrates (mammals, birds, turtles, alligators, amphibians) and invertebrates (Drosophila, hymenoptera, crustaceans, mollusks [16].

DMRT1 gene (Doublesex and Mab-3-Related Transcription factor $\underline{1}$ ) is localized in Z chromosome in birds. It is expressed in the developing embryo in the urogenital system as well as in mice. Its ortholog was identified in short arm chromosome 9 humans and it is responsible for the appearance of individuals with chromosomal inversion of gender. It is related dsx (doublesex) of Drosophila and mab3 (male abnomal 3) of nematode (Caenorhabditis elegans). The proteins encoded by these genes contain DNA-binding motif (DM-domain). In mice Dmrt1 gene is expressed exclusively in gonadal tubercle in the XX and XY embryos and then with the development in the seminiferous tubules, testes, Sertoli cells, germ cells. Violating of its activity gives rise to XY mice with defective testes.

In chick expression associated with medulla is intense in the gonads of males and appears at 4.5 - 5.5 day (stages 25 - 28) of development and even at 3.5 days of development (stage 22). This gene is expressed in gonadal strip of birds (for $S R Y$ is only typical) during its formation and generation of Wolffian ducts. Its activity is more high in males' embryos than in females. DMRT1 gene is located at the site of $\mathrm{Z}$ chromosome cytogenetically coinciding with MHM region and hence may be supposed to play the role in dosage compensation and sex determination in birds. DMRT1 gene is expressed at day 3 of development and the differentiation of sex to the histological level begins on day 6 of embryonic development. RNA interference mediated suppression of DMRT1 leads to feminization of the testis at both structural and molecular level. It should be noted that sexual dimorphism in gene expression of the birds occurs before histological differentiation of the gonads. DMRT1 is strongly expressed in males at 3.5-day of development (22 stage) for morphologically undifferentiated gonads. $A M H$ is also working on stages 25 - 28 before differentiation. At the molecular level differentiation of bird gonads occurs earlier than morphological. So far it is not clear what key genes regulate (aromatase and $A M H$ ) sex determination [3] [17].

Shetty et al. (2002) have cloned part of the candidate bird sex-determining gene DMRT1 from the emu and have shown that it is expressed during the stages of development corresponding to gonadal differentiation in the chicken. The gene maps to the distal region of the $\mathrm{Z}$ short arm and is absent from the large $\mathrm{W}$ chromosome. Because most sequences on the emu $\mathrm{W}$ chromosome are shared with the $\mathrm{Z}$, the Z-specific location constitutes strong evidence that differential dosage of DMRT1 is involved in sex determination in all birds. The sequence of emu DMRT1 gene has 88\% homology with chicken DMRT1 and 65\% with human DMRT1. Unexpectedly, an unexpressed 270-bp region in intron 3 of emu DMRT1 gene showed $90 \%$ homology with a sequence in the corresponding intron of human DMRT1 [18]. This high conservatism suggests important role in controlling the expression of DMRT1, for example, control of methylation in females. Two copies of the gene are likely required for the appearance of testes in ZZ males [18]. DMRT1 gene is strongly expressed in males at the early stages of development (stage 22) and can be a dose-Z factor in the differentiation of sex. The usage of impact of an aromatase inhibitor like fadrosole on ZW embryos gives an opportunity to demonstrate that here is a significant increase in the activity of the DMRT1 gene compared with SOX9 gene as in ZZ individuals. It is assumed that the development of testes do not need two doses of DMRT1, no matter the difference in the dose and the activity of this gene is necessary for the differentiation of testes. Turning this gene by RNA interference in ZZ embryos leads to the feminization of the gonads and the evidence in favor of the hypothesis of $\mathrm{Z}$ dose of sex determination in chickens. Interestingly a conserved region of 180 bp TESCO (ECR-evolutionary conserved region) was described in the birds as well as in the monotremes, marsupials, reptiles, amphibians. It described a conserved region of 180 bp TESCO part of an enhancer responsible for the activation of Sox 9 in mammals. It is assumed that in vertebrates expression of SOX9 governed through ECR with SOX, TCF/LEF, Forkhead, DMRT1, GATA protein. For birds such role plays DMRT1. This gene is expressed in birds prior to Sox9 and probably regulates its expression like $S R Y$ [19]. The appearance of testes still depends on AMH (antimullerian hormone). AMH gene expressed in both sexes at a low level but its activity is increased in males during differentiation of the gonads. It is assumed that its activation is independent of the SOX9 gene (Figure 4). Most recently, another Z-linked male factor has been identified, Hemogen (HEMGN), which is involved in testicular differentiation in the chicken embryo. Over-expression of HEMGN in female embryos can cause elevation of both DMRT1 and SOX9 expression, together with down-regulation of FOXL2 and aromatase [20]. However it was shown that over-expression of DMRT1 induceds the male pathway genes and antagonizes the female pathway in embryonic chicken gonads. Ectopic DMRT1 expression in female gonads induces SOX9 and AMH expression. It also induces expression of the Z-linked male factor Hemogen (HEMGN). Furthermore, expression of the critical femi- 
nizing enzyme, aromatase, is reduced in the presence of over-expressed DMRT1. These data indicate that DMRT1 is an essential sex-linked regulator of gonadal differentiation and that it likely acts via a dosage mechanism established through the lack of global $\mathrm{Z}$ dosage compensation in birds. Several promoters were analyzed and it was found that although the SF1 promoter produced a tissue-restricted expression pattern that was the highest in the mesonephros and liver, it was also higher in the gonads compared to the rest of the body [21] [22].

There is a model of bird sex determination, potentially including DMRT1, involves direct interaction between a W-derived factor (Factor F) and the Z-chromosome. Here the W-encoded factors mediate the expression of Z chromosome genes (for example, DMRT1). The neighboring MHM area, containing $2.2 \mathrm{~kb}$ tandem repeat, is hypermethylated in both chromosomes of males and hypomethylated in the same one female region of $\mathrm{Z}$. W chromosome is required for hypomethylation. In triploid 3A:ZZZ MHM region is not active and in 3A:ZZW individuals both $\mathrm{Z}$ transcribed as $\mathrm{W}$ is present. RNA-seq was used to produce a comprehensive profile of gene expression in chicken blastoderm and embryonic gonads prior to sexual differentiation. The robust sexually dimorphic gene expression in both tissues pre-dating gonadogenesis, including sex-linked and autosomal genes, was found. These data support the hypothesis that sexual differentiation at the molecular level is at least partly cell autonomous in birds. It is possible that additional Z-linked genes may play a role in sexual differentiation in different tissues through changes in their relative expression [23].

Although birds diverged from reptiles approximately 100 MYA, the ovary-determining action of estrogens remains present in birds with the evolution of a genetic mechanism for sex determination (Figure 4), [24]-[26]. According to Pask A.J. [25] in the presence of estrogens key male differentiation genes fail to be up regulated in the XY gonad and instead key ovary-promoting genes are up regulated leading to ovarian development. The gonad development is sensitive to the manipulation with hormones. Two enzymes- $\mathrm{P} 450$ aromatase, $17 \beta$-HSD —are required for the synthesis of estrogens only expressed in ZW gonads in the stage of morphological resolution (6 6.5 days, stage 29 - 30). The females of 3 - 4 days of embryonic development develop testes or ovotestis after degenerative diseases of the ovaries, polyploidy, left ovariectomy, extraembryonic testis grafting, hypertrophy of the right-gonads, implanted testes of 13-day-old etc. Interestingly the males in the chick embryos are heavier than females, and the anti-androgen injection on day 8 of incubation reduces the difference. Castration, transplantation, injection of estrogen, anti-estrogens, aromatase inhibitors can alter sex in birds, but the results are difficult to interpret in the sense of the primary sex determination [25]. Experimentally induced female-to-male sex reversal using the aromatase inhibitor fadrozole showed elevated levels of DMRT1 expression similar to those of normal males (with two copies of the $\mathrm{Z}$ chromosome). It implies that the gene involvement in male development [27]. Aromatase inhibitor also reduced expression of FOXL2 in the embryonic chicken ovary [28]. Although sex-reversed chickens have developed testes containing essentially the same cellular components as those of normal male testes and were capable of complete spermatogenesis the limitations encountered are low sperm counts and blind-ended has deferens production. So, oestrogen obviously plays a key role in the gonadal differentiation in birds and genetically female chicken embryos can develop testes and a male phenotype.

In birds, especially chicken Gallus gallus domesticus there were described numerous examples of the sex reversal by injection of estrogen, inhibitors of its metabolism, removal of the gonads [26] [28]. In recent years, epigenetic mechanisms of such alterations have been studied [26] [28]. Unlike mammals, which initiate the biosynthesis of steroids in the gonads only after the sexual differentiation, birds estrogen synthesis begins in the female undifferentiated embryonic gonads that are sensitive to hormonal manipulation. For the terminal step estrogen biosynthesis requires two enzyme, $17 \beta$-HSD and aromatase, which are synthesized immediately prior to differentiation in the ovary of 6.0 - 6.5 days of embryonic development. FOXL2 gene activity coincides with the expression of these two enzymes [26]. For this object it was demonstrated control of gene activity by means of aromatase cyp19a1 site-specific methylation. It was shown that DNA methylation within the 170 bp promoter region on chromosome in CpG (-955), CpG (-869), CpG (-789) that reflected phenotypic sexual differentiation on day 19 of embryonic development and feminization of embryonic gonads. The protocol was developed to quantify feminization of embryonic gonads ZZ-males when injected ethynylestradiol in the yolk of eggs at different stages of development. It was demonstrated the existence of "epigenetic markers of sex" in individuals with inversion of sex after exposure to estrogen. It is believed that these epigenetic markers are able to overcome the effect of DMRT1 on 3.5 days of development and anti-Mullerian hormone on day 5, but not Sox9. It was shown even greater stability of epigenetic sex compared to morphological one. Gonads of birds on stage injection EE2-3 - 4 days incubation contain somatic cells and PGC of future gonads of both types. At the same time 
estrogen affects only somatic cells but does not affect predecessor of germ one [29]. So the control of aromatase activity through its methylation in chickens and the impact on estrogen metabolites can significantly reduce the activity of at least the main male sex determining genes and cause temporary inversion of gender different from the complete inversion of fish or reptiles.

There is speculation about the presence in birds other sex determination mechanism of control associated with the synthesis of specific non-coding RNA and selective shutdown female gene alleles DMRT1. It is able to show that on day 1 after fertilization set differential methylation status of the region of the genome MHM (MaleHypermethylated). Female MHM is hypomethylated and synthesizes options noncoding RNA. It is important to note that the MHM coincides with the cytogenetic position of DMRT1 on $\mathrm{Z}$ chromosome which may allow the gene to work actively in males. Articles of Melamed E. and Arnold A. [30] who assessed regional differences in dosage compensation on the $\mathrm{Z}$ chromosome demonstrated the role of part $\mathrm{Zp}$ ( $\mathrm{Z}$ valley) localized cluster of compensated genes. In male MHM locus is hypermethylated and inactivated in the sense RNA synthesis, and therefore both alleles DMRT1 are not transcribed. MHM locus consists of approximately 200 repeats of a sequence $2.2 \mathrm{~kb}$ and transcribed into noncoding heterogeneous RNA, which accumulates in the nucleus of female locally to the site of transcription [31]. It was demonstrated specific chromatin modification for females near MHM l. Chromatin chickens Zp21 site, including the locus MHM, enriched histone acetylation of H4 lysine 16 in females but not in males [32]. Demethylating agent 5-aza-cytidine has an asymmetric effect on both Z chromosomes of males, changing the configuration of chromatin, RNA expression MHM, and modification H4K16Ac, assuming inequality in chromatin methylation status of both $\mathrm{Z}$ chromosomes. Interestingly, that MHM site was described for turkey and other species [33]. It is worth noting that the zebra finch (Taeniopygia guttata) noncoding RNA missed association with MHM and the corresponding portion of a special regulation of dosage compensation [34]. Injection of plasmid with cMHM in testes of adult males leads to a significant weakening of expression of DMRT1 and increase such estrogen ER [35]. The participation of the MHM sense and antisense transcripts in the regulation of early embryogenesis and gametogenesis of chickens, and in particular effects on DMRT1 was confirmed experimentally after induction of sex reversal in chickens with aromatase inhibitor [36]. Modified cMHM amplification has the lowest level of methylation in the ovaries, and the highest one in conventional male. Methylation was higher in the left testis of sex inverted females than in the left ovotestis weaker sex inverted animals [37]. In a recent study of brazilian geneticists that used variant of RT-PCR was shown that the largest transcription of MHM for the analyzed period of incubation (4 - 14 days) is detected at 8 and 14. The males in the absence of transcription of this gene show an intense activity of DMRT1 [38]. Results of work once again confirm the participation of RNA MHM in differential regulation of male and female alleles of DMRT1 gene. However, the question is raised about the possible contribution to the determination some factors involved in the earlier stages gonadogenesis. According to our preliminary data processing chick embryos on day 1 of incubation with 5-aza-cytidine leads to the transformation of the gonads of males in females and even entry of germ cells in meiosis. For chickens there was described certain number of short size miRNA of 20 - 24 bp arising from 70 - 80 bp pre-miRNAs (miR-202*, miR-363, miR-101, miR-31, miR-206) evinced differential expression during gonadogenesis though the exact role has not been established [39] [40].

\section{References}

[1] Ellegren, H. (2011) Emergence of Male-Biased Genes on the Chicken Z-Chromosome: Sex-Chromosome Contrasts between Male and Female Heterogametic Systems. Genome Research, 21, 2082-2086. http://dx.doi.org/10.1101/gr.119065.110

[2] Gamble, T. and Zarkower, D. (2012) Sex Determination. Current Biology, 22, R257-R262. http://dx.doi.org/10.1016/j.cub.2012.02.054

[3] Smith, C.A. (2010) Sex Determination in Birds: A Review. Emu, 110, 364-377. http://dx.doi.org/10.1071/MU10030

[4] Nanda, I., Haaf, T., Schartl, M., Schmid, M. and Burt, D.W. (2002) Comparative Mapping of Z-Orthologous Genes in Vertebrates: Implications for the Evolution of Avian Sex Chromosomes. Cytogenetic Genome Research, 99, 178-184. http://dx.doi.org/10.1159/000071591

[5] Ayers, K.L., Smith, C.A. and Lambeth, L.S. (2013) The Molecular Genetics of Avian Sex Determination and Its Manipulation. Genesis, 51, 325-336. http://dx.doi.org/10.1002/dvg.22382

[6] Chen, N., Bellott, D.W., Page, D.C. and Clark, A.G. (2012) Identification of Avian W-Linked Contigs by Short-Read Sequencing. BMC Genomics, 13, 183. http://dx.doi.org/10.1186/1471-2164-13-183

[7] (2011) Consortium ICG: Gallus gallus 4.0. 
[8] Rodrigue, K.L., May, B.P., Famula, T.R. and Delany, M.E. (2005) Meiotic Instability of Chicken Ultra-Long Telomeres and Mapping of a 2.8 Megabase Array to the W-Sex Chromosome. Chromosome Research, 13, 581-591. http://dx.doi.org/10.1007/s10577-005-0984-7

[9] Ayers, K.L., Davidson, N.M., Demiyah, D., Roeszler, K.N., Grützner, F., Sinclair, A.H., Oshlack, A. and Smith C.A. (2013) RNA Sequencing Reveals Sexually Dimorphic Gene Expression before Gonadal Differentiation in Chicken and Allows Comprehensive Annotation of the W-Chromosome. Genome Biology, 14, R26. http://dx.doi.org/10.1186/gb-2013-14-3-r26

[10] Wright, A.E., Moghadam, H.K. and Mank, J.E. (2012) Trade-Off between Selection for Dosage Compensation and Masculinization on the Avian Z Chromosome. Genetics, 192, 1433-1445. http://dx.doi.org/10.1534/genetics.112.145102

[11] Vicoso, B., Kaiser, V.B. and Bachtrog, D. (2013) Sex-Biased Gene Expression at Homomorphic Sex Chromosomes in Emus and Its Implication for Sex Chromosome Evolution. Proceedings of the National Academy of Sciences of the United States of America, 110, 6453-6458. http://dx.doi.org/10.1073/pnas.1217027110

[12] Lambeth, L.S. and Smith, C.A. (2012) Disorders of Sexual Development in Poultry. Sex Development, 6, 96-103. http://dx.doi.org/10.1159/000334059

[13] Lin, M., Thorne, M.H., Martin, I.C., Sheldon, B.L. and Jones, R.C. (1995) Development of the Gonads in the Triploid (ZZW and ZZZ) Fowl, Gallus Domesticus and Comparison with Normal Diploid Males (ZZ) and Females (ZW). Reproduction, Fertility and Development, 7, 1185-1197. http://dx.doi.org/10.1071/RD9951185

[14] Smith, C.A., Roeszler, K.N., Ohnesorg, T., Cummins, D.M., Farlie, P.G., Doran, T.J. and Sinclair, A.H. (2009) The Avian Z-Linked Gene DMRT1 Is Required for Male Sex Determination in the Chicken. Nature, 461, 267-271. http://dx.doi.org/10.1038/nature08298

[15] She, Z.Y. and Yang, W.X. (2014) Molecular Mechanisms Involved in Mammalian Primary Sex Determination. Journal of Molecular Endocrinology, 53, R21-R37. http://dx.doi.org/10.1530/JME-14-0018

[16] Bachtrog, D., Mank, J.E., Peichel, C.L., Kirkpatrick, M., Otto, S.P., Ashman, T.L., Hahn, M.W., Kitano, J., Mayrose, I., Ming, R., Perrin, N., Ross, L., Valenzuela, N. and Vamosi, J.C. (2014) Sex Determination: Why So Many Ways of Doing It? PLoS Biology, 12, e1001899. http://dx.doi.org/10.1371/journal.pbio.1001899

[17] Chue, J. and Smith, C.A. (2011) Sex Determination and Sexual Differentiation in the Avian Model. FEBS Journal, 278, 1027-1034. http://dx.doi.org/10.1111/j.1742-4658.2011.08032.x

[18] Shetty, S., Kirby, P., Zarkower, D. and Graves, J.A. (2002) DMRT1 in a Ratite Bird: Evidence for a Role in Sex Determination and Discovery of a Putative Regulatory Element. Cytogenetic Genome Research, 99, 245-251. http://dx.doi.org/10.1159/000071600

[19] Bagheri-Fam, S., Sinclair, A.H., Koopman, P. and Harley, V.R. (2010) Conserved Regulatory Modules in the Sox9 Testis-Specific Enhancer Predict Roles for SOX, TCF/LEF, Forkhead, DMRT and GATA Proteins in Vertebrate Sex Determination. The International Journal of Biochemistry and Cell Biology, 42, 472-477. http://dx.doi.org/10.1016/j.biocel.2009.07.001

[20] Nakata, T., Ishiguro, M., Aduma, N., Izumi, H. and Kuroiw, A. (2013) Chicken Hemogen Homolog Is Involved in the Chicken-Specific Sex-Determining Mechanism. Proceedings of the National Academy of Sciences of the United States of America, 110, 3417-3422. http://dx.doi.org/10.1073/pnas.1218714110

[21] Lambeth, L.S., Raymond, C.S., Roeszler, K.N., Kuroiwa, A., Nakata, T., Zarkower, D. and Smith C.A. (2014) OverExpression of DMRT1 Induces the Male Pathway in Embryonic Chicken Gonads. Developmental Biology, 389, 160172. http://dx.doi.org/10.1016/j.ydbio.2014.02.012

[22] Lambeth, L.S., Ohnesorg, T., Cummins, D.M., Sinclair, A.H. and Smith, C.A. (2014) Development of Retroviral Vectors for Tissue-Restricted Expression in Chicken Embryonic Gonads. PLOS ONE, 9, e101811. http://dx.doi.org/10.1371/journal.pone.0101811

[23] Smith, C.A. and Sinclair, A.H. (2004) Sex Determination: Insights from the Chicken. Bioessays, 26, 120-132. http://dx.doi.org/10.1002/bies.10400

[24] Ditewig, A.C. and Yao, H.H. (2005) Organogenesis of the Ovary: A Comparative Review on Vertebrate Ovary Formation. Organogenesis, 2, 36-41. http://dx.doi.org/10.4161/org.2.2.2491

[25] Pask, A.J. (2012) A Role for Estrogen in Somatic Cell Fate of the Mammalian Gonad. Chromosome Research, 20, 239245. http://dx.doi.org/10.1007/s10577-011-9260-1

[26] Trukhina, A.V., Lukina, N.A., Wackerow-Kouzova, N.D. and Smirnov, A.F. (2013) The Variety of Vertebrate Mechanisms of Sex Determination. Biomed Research International, Current Advances in Molecular Phylogenetics, 2013, Article ID: 587460. http://dx.doi.org/10.1155/2013/587460

[27] Smith, C.A., Katz, M. and Sinclair, A.H. (2003) DMRT1 Is Upregulated in the Gonads during Female-to-Male Sex Reversal in ZW Chicken Embryos. Biology of Reproduction, 68, 560-570. 
http://dx.doi.org/10.1095/biolreprod.102.007294

[28] Hudson, Q.J., Smith, C.A. and Sinclair, A.H. (2005) Aromatase Inhibition Reduces Expression of FOXL2 in the Embryonic Chicken Ovary. Developmental Dynamics, 233, 1052-1055. http://dx.doi.org/10.1002/dvdy.20388

[29] Ellis, H.L., Shioda, K., Rosenthal, N.F., Coser, K.R. and Shioda, T. (2012) Masculine Epigenetic Sex Marks of the CYP19A1/Aromatase Promoter in Genetically Male Chicken Embryonic Gonads Are Resistant to Estrogen-Induced Phenotypic Sex Conversion. Biology of Reproduction, 87, 1-12.

[30] Melamed, E. and Arnold, A.P. (2007) Regional Differences in Dosage Compensation on the Chicken Z Chromosome. Genome Biology, 8, R202. http://dx.doi.org/10.1186/gb-2007-8-9-r202

[31] Teranishi, M., Shimada, Y., Hori, T., Nakabayashi, O., Kikuchi, T., Macleod, T., Pym, R., Sheldon, B., Solovei, I., Macgregor, H. and Mizuno, S. (2001) Transcripts of the MHM Region on the Chicken Z Chromosome Accumulate as Non-Coding RNA in the Nucleus of Female Cells Adjacent to the DMRT1 Locus. Chromosome Research, 9, 147-165. http://dx.doi.org/10.1023/A:1009235120741

[32] Bisoni, L., Batlle-Morera, L., Bird, A.P., Suzuki, M. and McQueen, H.A. (2005) Female-Specific Hyperacetylation of Histone H4 in the Chicken Z Chromosome. Chromosome Research, 13, 205-214. http://dx.doi.org/10.1007/s10577-005-1505-4

[33] Itoh, Y., Kampf, K. and Arnold, A.P. (2011) Possible Differences in the Two Z Chromosomes in Male Chickens and Evolution of MHM Sequences in Galliformes. Chromosoma, 120, 587-598. http://dx.doi.org/10.1007/s00412-011-0333-x

[34] Itoh, Y., Replogle, K., Kim, Y.H., Wade, J., Clayton, D.F. and Arnold, A.P. (2010) Sex Bias and Dosage Compensation in the Zebra Finch versus Chicken Genomes: General and Specialized Patterns among Birds. Genome Research, 20, 512-518. http://dx.doi.org/10.1101/gr.102343.109

[35] Yang, X., Zheng, J., Xu, G., Qu, L., Chen, S., Li, J. and Yang, N. (2010) Exogenous cMHM Regulates the Expression of DMRT1 and ER Alpha in Avian Testes. Molecular Biology Report, 37, 1841-1847. http://dx.doi.org/10.1007/s11033-009-9619-y

[36] Roeszler, K.N., Itman, C., Sinclair, A.H. and Smith, C.A. (2012) The Long Non-Coding RNA, MHM, Plays a Role in Chicken Embryonic Development, including Gonadogenesis. Developmental Biology, 366, 317-326. http://dx.doi.org/10.1016/j.ydbio.2012.03.025

[37] Yang, X., Zheng, J., Qu, L., Chen, S., Li, J., Xu, G. and Yang, N. (2011) Methylation Status of cMHM and Expression of Sex-Specific Genes in Adult Sex-Reversed Female Chickens. Sexual Development, 5, 147-154. http://dx.doi.org/10.1159/000327712

[38] Caetano, L.C., Gennaro, F.G.O., Coelho, K., Araújo, F.M., Vila, R.A., Araújo, A., de Melo Bernardo, A., Marcondes, C.R., Chuva de Sousa Lopes, S.M. and Ramos, E.S. (2014) Differential Expression of the MHM Region and of Sex-Determining-Related Genes during Gonadal Development in Chicken Embryos. Genetics and Molecular Research, 13, 838-849. http://dx.doi.org/10.4238/2014.February.13.2

[39] Cutting, A.D., Bannister, S.C., Doran, T.J., Sinclair, A.H., Tizard, M.V. and Smith, C.A. (2012) The Potential Role of MicroRNAs in Regulating Gonadal Sex Differentiation in the Chicken Embryo. Chromosome Research, 20, 201-213. http://dx.doi.org/10.1007/s10577-011-9263-y

[40] Feng, Y.P., Chen, J.F., Huang, P., Wang, X., Wang, J., Peng, X.L. and Gong, Y.Z. (2014) Expression Analysis of Differentially Expressed MIRNAs in Male and Female Chicken Embryos. Genetics and Molecular Research, 13, 30603068. http://dx.doi.org/10.4238/2014.April.17.2 
Scientific Research Publishing (SCIRP) is one of the largest Open Access journal publishers. It is currently publishing more than 200 open access, online, peer-reviewed journals covering a wide range of academic disciplines. SCIRP serves the worldwide academic communities and contributes to the progress and application of science with its publication.

Other selected journals from SCIRP are listed as below. Submit your manuscript to us via either submit@scirp.org or Online Submission Portal.
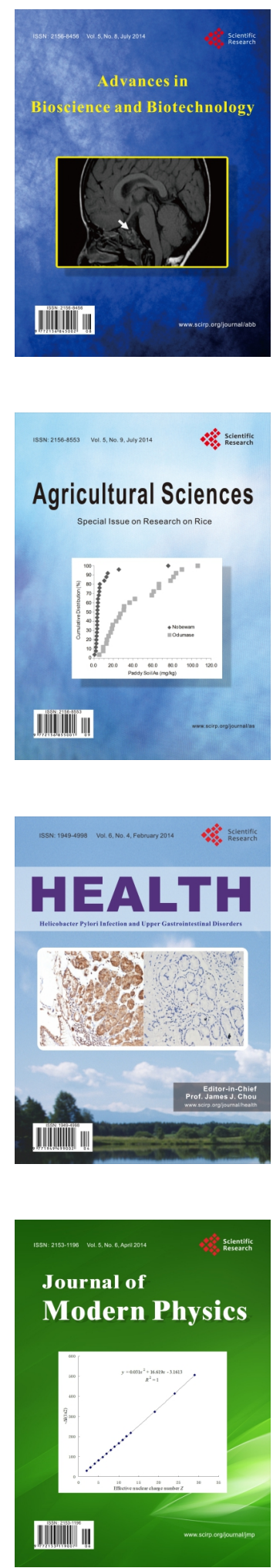
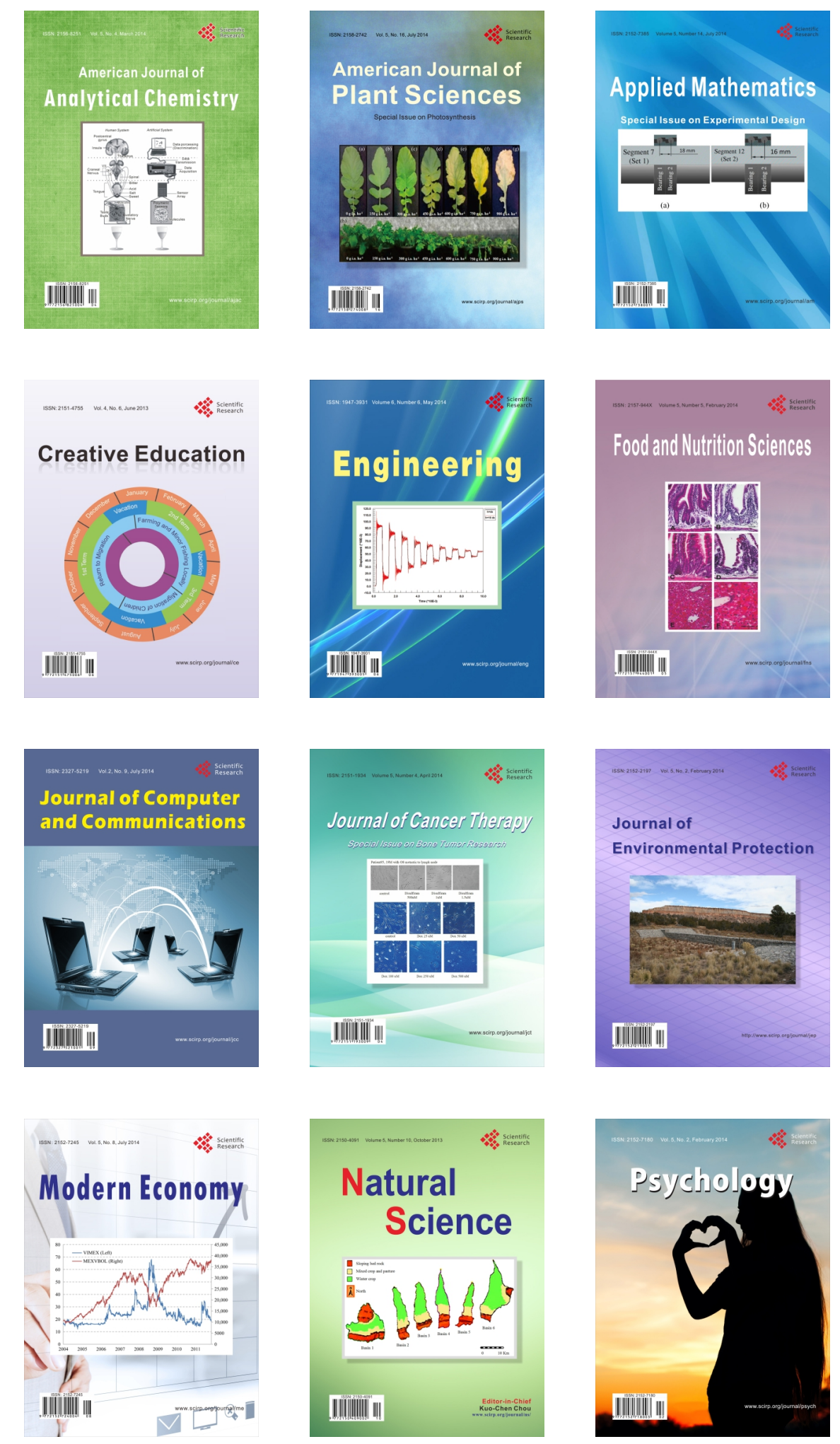